\section{P3.044 HIV RISK BEHAVIORS, IN JUVENILE DELINQUENTS AND IN FAMILY ABANDONMENT SITUATIONS. GUATEMALA, 2012}

doi:10.1136/sextrans-2013-051184.0504

'B Huamán, 'B Alvarez, 'I Loya-Montiel, 2 O Osuna-Ramírez, ${ }^{3} Z$ Pinzón, ${ }^{4} \mathrm{M}$ Mansur, ${ }^{4} \mathrm{~S}$ Ortega, "R Vega, 'S Morales-Miranda. 'Universidad del Valle de Guatemala, Guatemala, Guatemala, Universidad Autónoma de Sinaloa, Culiacán-Sinaloa, Mexico, ${ }^{3}$ Programa Nacional de sida, Ministerio de Salud, Guatemala, Guatemala, 4/nstituto Humanista de Cooperación para el Desarrollo, Guatemala, Guatemala

Background Juveniles who are exposed to a street life are at high risk for drug addiction, pregnancy, sexually transmitted infections (STI), among others due to their biological, psychological and social vulnerabilities.

Methodology A survey was conducted in Guatemala City; with the purpose to determine risk behaviours and HIV and syphilis prevalence among juvenile delinquents and in family abandonment situations. A stratified consecutive sampling method was used; a tablet was used to conduct a standardised face-to-face questionnaire, and both pre-consent from legal guardians as well as assent by the minor were required. HIV and Syphilis screenings were conducted. Proportions and confidence intervals (CI 95\%) adjusted for the sampling type were calculated using STATA 11.1.

Results 528 participants ages 15 to 17, 72\% male and 28\% female were enrolled. 58\% (ICR 50.1-64.9) began having sexual relations between 10 and 14 years old, 37\% (ICR 29.7-44.1) used a condom at first sexual act; only $24 \%$ (ICR 18.2-29.6) and 12\% (ICR 9.11-15.0) had access to free condoms and HIV tests in the past year. 81\% (ICR 75.7-85.8) reported currently being sexually active, 79\% (71.22$85.67)$ had a stable partner, $20 \%$ (13.71-28.14\%) occasional partner, and 1\% (0.14-1.54) commercial partner; 40\% (ICR: 32.8-47.9) reported using a condom a last sexual act. Out of the women surveyed, $16 \%(9.75-25.19)$ had been pregnant at one point and $53 \%$ of these had had an abortion. 57\% (50-67.7) had consumed illicit drugs; marijuana making up 93\% (ICR 87.8-96.6) and cocaine made up 31\% (ICR 23.7-40.1) topping the list. HIV and Syphilis prevalences were lower than $1 \%$.

Conclusions High pregnancy and illicit drug use proportions were reported, as well as low HIV and syphilis prevalences. Strengthening HIV prevention interventions, with an integral focus on reproductive sexual health adapted to this life stage and street context of this population, is necessary.

\section{P3.045 EPIDEMIOLOGICAL SURVEY OF HIV/AIDS PATIENTS IN ISFAHAN PROVINCE (IRAN) 1985 - 2012}

doi:10.1136/sextrans-2013-051184.0505

M Meshkati. Province Health Centre, Isfahan, Iran

Introduction AIDS is a significant threat for women. More than $50 \%$ of HIV infected people in the world are among women because the most common transmission way is sexual contacts. In IRAN sharing needle and syringe in IDUs is the most common way. Increasing number of HIV infection especially in the east Mediterranean countries as Iran should be investigated. So we looked HIV/ AIDS patients for managing HIV/AID control programme.

Methods In Isfahan, we performed a survey (cross - sectional) for all recorded HIV-positive cases in 1985 - 2012. The data was gathered by questionnaires.

Results Until January 2012, 492 HIV/AIDS cases have been recognised. The majority of cases are male (89\%) and in age group 25-34 $(69 \%) .44 \%$ of them are singles and $38 \%$ of them are married. In male, the most common transmission route is sharing needle and syringe ( $81 \%$ ) and in women is sexual contact (79\%).57(11\%) HIV infected women have been recognised that in $54 \%$ of them, their husbands were HIV positive that most of them were IDUs. We have 4 children under 15 years old that their parents are HIV positive.
Conclusions The number of infected women has increased (2 years ago in same time the number of infected women was lower, $5 \%$ of total cases). So we faced risk of third episode of HIV/AIDS from IDUs to sexual contact in Iran right now. The majority of women were infected by their husband. In result we must notice bridge community and their role for spreading HIV infection in general population. It is vital that women be noticed as leader of HIV/ AIDS campaign. We need to upgrade prevention programmes like training, counselling and harm reduction services for vulnerable groups especially women.

\section{P3.046 PROFILE OF WOMEN WITH HIV IN PERU}

doi:10.1136/sextrans-2013-051184.0506

S La Rosa, M Lazo, P Mallma, C Cárcamo, P García. Universidad Peruana Cayetano Heredia, Lima, Peru

Introduction The HIV epidemic in Peru, a continuing and important public health problem, has been concentrated in MSM from its outset. Despite advances in prevention and treatment of HIV, the epidemic in Peru has come to affect a growing proportion of women, who nowadays represent 1 of every 3 AIDS cases. In order to develop effective strategies for preventing HIV in women, it is important to know the profile of women who become infected.

Methods From November through December of 2011, we interviewd women ages 15-49 who had been diagnosed with AIDS within the previous 2.5 years, and who had received care at one of the largest 10 hospitals in Lima, Callao and Iquitos. The questionnaire was completing using EpiSurveyor in mobile phones, and contained demographic and risk behaviour questions.

Results Of 322 women contacted for the study, 321 (99.7\%) answered the questionnaire. The average age was 30 (range 16-49), $61 \%$ had a secondary education, $54 \%$ were cohabiting with their partners, $61.4 \%$ were housewives or unemployed, and $49.5 \%$ had monthly family incomes $\leq 200$ USD. The median age at sexual debut was 16 (range 7-25), the median lifetime number of partners was 3 (range 1-17), 33\% had had anal intercourse with their last partner, $41 \%$ knew that their most recent partner had had intercourse with other partners, $82.8 \%$ used a condom in their last sexual encounter, $39 \%$ had reported an STI in the previous 12 months, and $12.8 \%$ had been diagnosed with tuberculosis.

Conclusion Unlike previous reports, the profile of women with HIV in this study reflects high risk arising from her own sexual behaviour.

\section{P3.047 ONCOGENIC HPV PREVALENCE IN HIV INFECTED WOMEN ASSISTED IN PUBLIC HEALTH IN BUENOS AIRES (CABA)}

doi:10.1136/sextrans-2013-051184.0507

S V Vulcano, ${ }^{2,1} \mathrm{~F}$ González, ${ }^{2} \mathrm{M}$ Nan, ${ }^{2} \mathrm{~F}$ Portnoy, ${ }^{2} \mathrm{~A}$ Duran, ${ }^{2} \mathrm{C}$ abanillas G Fernandez Bouzas, 'MB HPV study grou. Coordinación SIDA, CABA, Buenos Aires, Argentina

Background The scientific evidence shows that HIV-infected women have increased risk of precancerous lesions than uninfected women. The objective was search the prevalence of HPV and assess the cyto-colposcopic and Hybrid Capture test correlation for highrisk HPV in HIV infected women in CABA.

Methods 269 HIV infected women over 18, not pregnant and without IGT lesions were included between October 2010 to July 2012. Clinical and gynaecological chart were reviewed and sampling for PAP, colposcopy and screening of high-risk HPV were performed. The detection of high-risk HPV was performed with the hybrid capture assay (Digene)

Results Prevalence of HPV and epidemiological profile were available in the 269 women.

$45 \%$ had HIV VL below 50 copies and $80 \%$ had CD4 upper than 350 cells/cc. 
A PAP in the last year before this study was available in $30 \%$ of cases.

The correlation of screening techniques with the three methods proposed: diagnostic cytology, capture hybrid and colposcopy was made in 169 women

Prevalence of HPV infection was 39\% (104 positive/165 negative).

Correlation between Cyto-colposcopic and Hybrid Capture test was:

hybrid capture $(-)$ cytology/colposcopy $(+)=2 / 169$

hybrid capture $(+)$ cytology/colposcopy $(-)=15 / 169$

hybrid capture $(+)$ cytology/colposcopy $=32 / 169$

hybrid Capture (-) cytology colposcopy $(-)=84 / 169$

hybrid Capture $(+)$ indeterminate cytology and colposcopy $(-)=19 / 169$

hybrid Capture (-) indeterminate cytology and colposcopy $(-)=17 / 169$

Conclusions Despite that it was not a large sample, the negative predictive value of hybrid captures for HPV detection was $98 \%$ in this population.

We recommend the incorporation of the hybrid capture as screening technique in all women with HIV regardless of the age and all those with negative cytology and positive hybrid capture for the early detection of cervical lesions.

\section{P3.048 HIV IN CHILDREN IN A GENERAL POPULATION SAMPLE IN SOUTHERN AFRICA: PREVALENCE, CAUSES AND EFFECTS}

doi:10.1136/sextrans-2013-051184.0508

'E L Pufall, 2,1C Nyamukapa, ' J W Eaton, ${ }^{2} \mathrm{R}$ Mutsindiri, ${ }^{2} \mathrm{G}$ Chawira, ${ }^{2} \mathrm{~S}$ Munyati, 'L Robertson, 'S Gregson. 'Imperial College London, London, UK; ${ }^{2}$ Biomedical Research and Training Institute, Harare, Zimbabwe

Background The epidemiology of HIV in children in sub-Saharan Africa remains poorly understood. To help address this gap we describe the prevalence, causes and effects of HIV in children (aged 2-14 years) and adolescents (aged 15-17 years) in a southern African population.

Methods General population survey of 3,390 children and 2,130 adolescents in Manicaland, eastern Zimbabwe. Data on possible socio-demographic correlates of HIV prevalence, sources of infection, and effects of HIV infection in children were analysed using multi-variable logistic regression. The contribution of horizontal transmission was assessed by investigating the survival and HIV infection status of mothers of infected children.

Results HIV prevalence was $2.15 \%(73 / 3,390)$ in children and $1.97 \%(42 / 2,130)$ in adolescents. Infection levels did not differ by sex, age, socio-economic status, location or religion. The mothers of most infected children were either deceased or HIV-positive which is consistent with MTCT being the primary mode of infection, but 9/73 infected children and 4/42 infected adolescents had surviving uninfected mothers. Infected children were more likely than uninfected children to be malnourished $(21.6 \%$ versus $9.9 \%, p=0.006)$ and stunted $(42.0 \%$ versus $30.5 \%, p=0.03)$. HIV infection was not correlated with physical or psychological ill-health in children, but infected adolescents were more likely to report episodes of illness than their uninfected counterparts $(p=0.003)$.

Conclusion Childhood HIV infection in Zimbabwe is due primarily to MTCT and is associated with poor physical development.

\section{P3.049 HIV/AIDS IN A PUERTO RICAN WOMEN POPULATION: PSYCHOSOCIAL, RISK BEHAVIOR AND LIFESTYLES}

doi:10.1136/sextrans-2013-051184.0509

D M Fernandez-Santos, A M Mayor, E Santiago, E Rios-Olivares, R F Hunter-Mellado. Universidad Central del Caribe, Bayamon, PR, United States
Background This study describes psychosocial, risk behaviour and lifestyle features of HIV/AIDS women in Bayamón, PR. It also compares the psychosocial, behavioural and lifestyle profile changes of this cohort according to their entry year to the registry (2000-2010). Methods Baseline data was analysed from a prospective survey of HIV/AIDS-infected women reported to the HIV Central Registry at the Universidad Central del Caribe, RCMI Program from 2000 to 2010. The study group was composed of 499 adult women, with AIDS or HIV infection reported upon arrival at the University Hospital Ramon Ruiz Arnau or the Bayamon Regional Immunology Clinic. Statistical analysis included frequencies, percentages, Chisquare, Chi Square for linear trend and Fisher Exact test.

Results A large proportion of women were under 46 years $(71.4 \%)$. Most reported not having graduate studies (98.8\%), being unemployed (70.9\%), not having a partner in the last year (57.0\%) and having children $(88.0 \%)$. The most frequent sexual risk behaviour was heterosexual activity (98.8\%), where $71.3 \%$ reported heterosexual activity in the last year and $14.4 \%$ reported prostitution activity. Risky lifestyles reported by women included: smoking tobacco $(64.7 \%)$, use of alcohol (38.3\%), use of psychoactive substances $(36.3 \%)$ and intravenous drug usage (IVDU) (36.3\%). Most frequent psychosocial factors reported were: episodes of depression (82.2\%), episodes of impulsivity (55.4\%), isolation (44.6\%), suicide attempt $(23.7 \%)$, time served in prison $(20.3 \%)$, voluntary miscarriage $(17.7 \%)$ and having been sexually assaulted (7.1\%). An increasing linear trend $(p<0.05)$ was observed among proportions of women that reported using psychoactive substances, attempting suicide and having been sexually assaulted.

Conclusions Women reported a wide spectrum of risky psychosocial, behavioural and lifestyle activities. Increasing changes among these were observed over the last decade. This effort was made possible by NIH grant number 8G12MD007583 and 8U54MD007587 from the National Institute on Minority Health and Heath Disparities.

\section{P3.050 HIGH-RISK UROGENITAL HPV INFECTIONS IN PARAMARIBO, SURINAME: PREVALENCE AND RISK FACTORS AMONG ETHNIC DIVERSE WOMEN}

doi:10.1136/sextrans-2013-051184.0510

IJ J van der Helm, ${ }^{2} \mathrm{D}$ Geraets, ${ }^{3} \mathrm{~A}$ Grunberg, ${ }^{2,4 \mathrm{~K}}$ Quint, ${ }^{5} \mathrm{~L}$ Sabajo, ${ }^{1,6,7 \mathrm{H}}$ de Vries. ${ }^{1}$ Health Service Amsterdam, Amsterdam, The Netherlands; ${ }^{2} D D L$ Diagnostic Laboratory, Rijswijk, The Netherlands; ${ }^{3}$ Dept. of Public Health Ministry of Health Suriname, Paramaribo, Suriname; ${ }^{4}$ Department of dermatology LUMC, University of Leiden, Leiden, The Netherlands; ${ }^{5}$ Dermatological Service, Ministry of Health Suriname, Paramaribo, Suriname; ${ }^{6}$ Academic Medical Center, University of Amsterdam, Amsterdam, The Netherlands; ${ }^{7}$ Centre for Infectious Disease Control, National Institute of Public Health and the Environment, Bilthoven, The Netherlands

Background and Aim: Cervical cancer is caused by high-risk (HR) Human Papilloma Virus (HPV) infections. The mortality rate of cervical cancer in Suriname is $8 / 100.000$ per year. Current HPV vaccinations protect against cancers induced by HR-HPV 16 and -18 , and are not yet available in Suriname. We estimated prevalence of, and risk factors for urogenital HR-HPV infections among women from five major ethnic groups in Paramaribo, Suriname in a pre-vaccination era.

Methods Between July 2009 and February 2010, women aged $\geq 18$ years were recruited at a Family Planning clinic and STI clinic. Vaginal swabs were collected and general HPV detection was performed using the highly sensitive broad-spectrum SPF10 PCR and DNA enzyme immunoassay (DEIA). Subsequently, DEIA-positive samples were genotyped by the LiPA25 reverse hybridization assay (Labo Bio-medical Products, The Netherlands), targeting 25 different genotypes. Logistic regression analysis was used to identify determinants of HPV infection. 